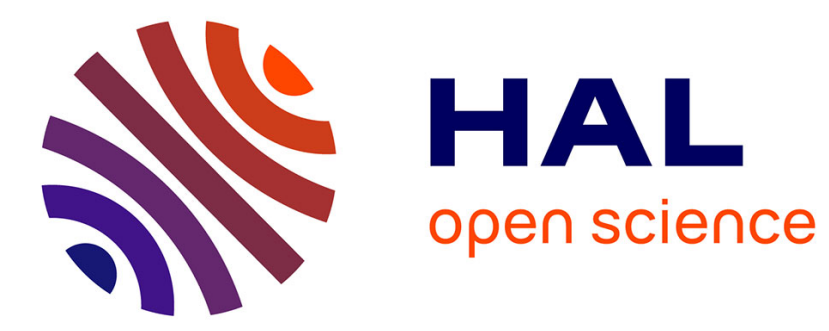

\title{
Superthermal light from single-mode VCSEL
}

T. Wang, D. Aktas, O. Alibart, É. Picholle, S. Tanzilli, G. P Puccioni, G. L

Lippi

\section{To cite this version:}

T. Wang, D. Aktas, O. Alibart, É. Picholle, S. Tanzilli, et al.. Superthermal light from single-mode VCSEL. Nonlinear Photonics, 2018, Zurich, Switzerland. NpM4C.4, 10.1364/NP.2018.NpM4C.4 . hal-02057735

\section{HAL Id: hal-02057735 \\ https://hal.science/hal-02057735}

Submitted on 5 Mar 2019

HAL is a multi-disciplinary open access archive for the deposit and dissemination of scientific research documents, whether they are published or not. The documents may come from teaching and research institutions in France or abroad, or from public or private research centers.
L'archive ouverte pluridisciplinaire HAL, est destinée au dépôt et à la diffusion de documents scientifiques de niveau recherche, publiés ou non, émanant des établissements d'enseignement et de recherche français ou étrangers, des laboratoires publics ou privés. 


\title{
Superthermal light from single-mode VCSEL
}

\author{
T. Wang ${ }^{1}$, D. Aktas ${ }^{2}$, O. Alibart, É. Picholle, S. Tanzilli \\ Université Côte d'Azur, INPHYNI, UMR 7010 CNRS, France \\ G.P. Puccioni \\ Istituto dei Sistemi Complessi, CNR, Via Madonna del Piano 10, I-50019 Sesto Fiorentino, Italy \\ G.L. Lippi \\ Université Côte d'Azur, INPHYNI, UMR 7010 CNRS, France \\ gian-luca.lippi@inphyni.cnrs.fr
}

\begin{abstract}
Light with superthermal properties is experimentally observed in the emission of a room-temperature, electrically-pumped single mode microcavity for pump values below continuous lasing. The observations are compared to Generalized Laser Rate Equations and stochastic simulations.
\end{abstract}

OCIS codes: $140.3945,270.5290$

Superthermal light has been recently recognized as a signature of Collective Effects (CE) in nanolasers since its origin is associated to the appearence of dipole-dipole correlations. The experimental observation of superthermal emission has been reported in the wings of emission pulses [1], accompanying the polarization switch [2,3], and in cw-pumped [4] microcavities. Thorough quantum-mechanical models, accompanied by numerical simulations, have also been proposed for systems with few emitters (and large coupling of the spontaneous emission into the lasing mode, i.e., $\beta \rightarrow 1$ ) $[1,4,5]$. A recent paper [6] proposes a set of Generalized Laser Rate Equations (GLREs) which includes the medium's polarization to characterize CEs in devices under more general conditions, i.e., independently of their $\beta$-factor, pump rate or relative relaxation rates (dipole vs. photon decay).

This approach is interesting since the standard adiabatic elimination, where the polarization variable is solved at equilibrium, is never entirely correct $[7,8]$. Thus, it stands to reason that even in those lasers - such as our microcavity - where the polarization decays much faster than the other variables (electromagnetic field and population inversion) a trace of its influence should survive. In this sense, the GLREs [6] represent a very interesting step forward for the description of small semiconductor-based devices (cf. [8] for a generalization of the simpler adiabatic elimination [7] to more realistic systems, including semiconductor lasers) and predict the occurrence of CEs in semiconductor lasers regardless of the limitations imposed by previous models $[1,4,5]$.

We report the experimental observation of superthermal light emitted by a single-mode microcavity (standard, electrically-pumped VCSEL) with a small fraction of spontaneous emission coupled into the lasing mode $(\beta \approx$ $\left.10^{-4}[9]\right)$ in the transition region from spontaneous emission to a coherent, lasing field. The laser is operated at room temperature under $\mathrm{cw}$ operation with a very good control of all the parameters (pump, environment, etc.) and above threshold emits on a single polarization mode with rejection ratio up to $24 \mathrm{~dB}$ (compared to the suppressed polarization). This strongly differentiates our observations from those already obtained in criogenically cooled, high- $\beta$ microcavities [2,3], where superthermal light emission is accompanied by polarization hopping.

Fig. 1 shows a temporal trace of the emission collected from a VCSEL-980 (Thorlabs) VCSEL by a fast (9.5 GHz bandwidth, Thorlabs PDA8GS) detector, sampled by a digital oscilloscope (LeCroy Wavemaster 8600) at $0.1 \mathrm{~ns}$ rate (6 $\mathrm{GHz}$ analog bandwidth). It shows a sequence of (small, due to the detector sensitivity) independent pulses - bunched light - emitted without any particular regularity (caracterised by a nearly monotonically decreasing radiofrequency power spectrum - cf. Fig. 3, top left panel, of the Supplementary Information accompanying [9]). The second-order, zero-delay autocorrelation $g^{(2)}(0)$ is obtained by equally splitting the laser output and detecting the coincidences with two single-photon detectors (APD idQuantique id100, $\approx 40 \mathrm{ps}$ jitter) using and AND gate with a Time-toAmplitude-Converter (Ortec 567, $\approx 15 \mathrm{ps}$ timing resolution). The value obtained for the autocorrelation is $g^{(2)}(0)=$ $2.15 \pm 0.05[10]$ in a regime where independent photon bunches are emitted, clearly indicating the superthermal nature of the emitted light.

Comparison to the theory [6] suggests compatibility with the influence of CEs in our system, since for the microcavity parameters (estimated to be $K \approx \times 10^{11} s^{-1}$ for the photon decay [9], $\gamma_{\perp} \approx \times 10^{13} s^{-1}$ for GaAs [11]) 


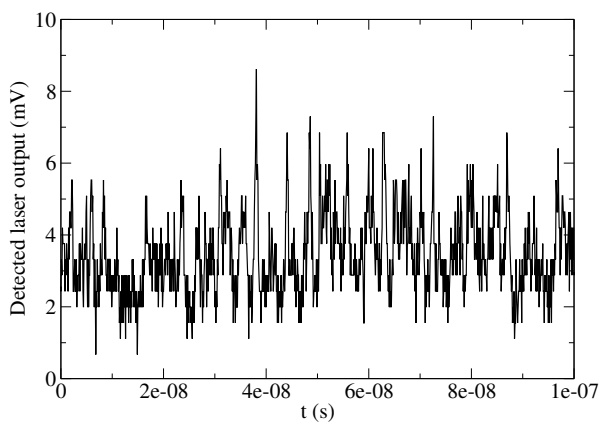

Fig. 1. Emission of bunched photons below the threshold for $\mathrm{cw}$ operation.

$g^{(2)}(0)$ values in excess of the thermal limit are expected (cf. Fig. 3 in [6]). The measured values are, however, larger than the limit estimated in [6] at $g^{(2)}(0) \lesssim 2.01$. On the other hand, such large values are compatible with stochastic simulations [12] which, based on Einstein's semiclassical theory of stimulated emission [13], do not include the dipole-dipole interactions. The stochastic simulations [12] predict a dynamical output of the same kind as observed (Fig. 1), thus suggesting that the photon bunching be of dynamical nature. At the present time, however, it is possible that the CEs may also contribute to the dynamics [6], thus strengthening the superthermal emission properties. These points will be the object of additional investigations.

${ }^{1}$ Currently at ...

${ }^{2}$ Currently at ...

\section{References}

1. F. Jahnke, Ch. Gies, M. Aßman, M. Bayer, H.A.M. Leymann, A. Foerster, J. Wiersig, Ch. Schneider, M. Kamp, and S. Höfling, "Giant photon bunching, superradiat pulse emission and excitation trapping in quantum-dot nanolasers", Nature Comm. 7, 11540 (2016).

2. Ch. Redlich, B. Lingnau, S. Holzinger, E. Schlottmann, S. Kreinberg, Ch. Schneider, M. Kamp, S. Höfling, J. Wolters, S. Reitzenstein, and K. Lüdge, "Mode-switching induced super-thermal bunching in quantum-dot microlasers", New J. Phys. 18, 063011 (2016).

3. E. Schlottmann, M. von Helversen, H.A.M. Leymann, Th. Lettau, F. Krüger, M. Schmidt, Ch. Schneider, M. Kamp, S. Höfling, J. Beyer, J. Wiesig, and S. Reitzenstein, "Exploring the Photon-Number Distribution of Bimodal Microlasers", arXiv:1709.04312v2 (2017).

4. S. Kreinberg, W.W. Chow, J. Wolters, Ch. Schneider, Ch. Gies, F. Jahnke, S. Höfling, M. Kamp, and S. Reitzenstein, "Emission from quantum-dot high-beta micocavities: transition from spontaneous emission to lasing and the effects of superradiant emitter coupling”, Light: Science \& Applications 6, e17030 (2017).

5. H.A.M. Leymann et al., "Sub- and superradiance in nanolasers", Phys. Rev. Appl. 4, 044018 (2015).

6. I.E. Protsenko, E.C. André, A.V. Uskov, J. Mørk, and M. Wubs, ”Collective Effects in Nanolasers Explained by Generalized Rate Equations", arXiv1709.08200v3 (2017).

7. G.L. Oppo and A. Politi, "Improved adiabatic elimination in laser equations", Europh. Lett. 1, 549-553 (1986).

8. G.L. Oppo and A. Politi, "Center-manifold reduction for laser equations with detuning", Phys. Rev. A 40, 1422-1427 (1989).

9. T. Wang, G.P. Puccioni, and G.L. Lippi, "Dynamical Buildup of Lasing in Mesoscale Devices", Sci. Rep. 5, 15858 (2015).

10. T. Wang, D. Aktas, O. Alibart, É. Picholle, G.P. Puccioni, S. Tanzilli, and G.L. Lippi, "Nontrivial photon statistics in small scale lasers", arXiv1710.02052v1 (2017).

11. L.A. Coldren and S.W. Corzine, Diode Lasers and Photonic Integrated Circuits, (Wiley, New York, 1995).

12. G.P. Puccioni and G.L. Lippi, "Stochastic Simulator for modeling the transition to lasing", Opt. Express 23, 2369-2374 (2015).

13. A. Einstein, "Zur Quantentheorie der Strahlung", Physikalische Zeitschr. 18, 121-128 (1917). 